\title{
Remarks on Duality Mapping and the Lax-Milgram Property
}

\author{
J. KoLomÝ
}

Es werden einige Bedingungen abgeleitèt, die sicherstellen, da $\beta$ die Dualitäts-Abbildung ein Homeomorphismus von einem Banach-Raum $X$ auf $X^{*}$ ist, 'der überdies auf einer dichten Teilmenge von $\dot{X}$ oberhalbstetig ist. Es werden ferner einige weitere Eigenschaften der Dualitäts-Abbildung bewiesen, die mit der Struktur des Banachraumes $X$ zusammenhängen, und es wird die sogenannte Lax-Milgram Eigenschaft der betrachteten Bilinearformen untersucht. .

Находятся условия, при которых дуальное отображение является гомеоморфизмом из Банахова простраиства $X$ на $\dot{X}^{*}$ и является полунепрерывным сверху на некотором піотном в $X$ множестве. Рассматриваются дальнейшие свойства дуального отображения в-связи с геометрической структурой Банах́ова пространств $X$ и свойство Лакс-Милграма билинейных форм.

Some conditions under which the duality map is a homeomorphism from a Banach space $X$ onto $X^{*}$ and upper-semicontinuous at some dense subset of $X$ are derived. Some further properties of the duality mapping are established in connection with the structure of the Banach space $X$. The so-called Lax-Milgram property of the bilinear forms is also investigated.'

\section{Introduction}

The concept of duality mapping introduced.independently by BEURLIVG and LIVINGSTON [2] and CoDra [4] has been used in several branches in functional analysis and its applications: theory of monotone and accretive operators, fixed point theory of nonexpansive (and related) operators, theory of approximations and geometry of Banach spaces.

Codra [4] proved that the duality mapping $J$ is always upper-senicontinuous on $X$ when $X$ has the norm and the dual space has the $\sigma\left(X^{*}, X\right)$-topology, while KENDERov [18] extended this result to maximal monotone operators. Upper-semicontinuity of duality mapping and subdifferential maps has been studied by GLES, GrEGory and Sims [13] and GREGORY [14], where upper-semicontinuity is characterized in terms of slices of the closed unit ball and upper-semicontinuity properties are related to the geometric structure of the spaces and properties of convex functions.

The purpose of this note is to derive some conditions under which the duality map $J$ is a honeomorphism of $X$ onto $X^{*}$ and upper-semicontinuous at the dense subset of the given space. Some further properties of the duality mapping are derived in connection with the structure of Banach spaces. Furthermore, so-called Lax-Milgram property of the bilinear forms is investigated. 


\section{Definitions and notations}

Let $X$ be a real normed linear space, $X^{*}$ its dual space, $\langle\cdot, \cdot\rangle$ the pairing between $X^{*}$ and $X$. Let $B_{1}(0), B_{1}^{*}(0), B_{1}^{* *}(0)$ denote the closed unit balls; $S_{1}(0) ; S_{1}^{*}(0), S_{1}^{* *}(0)$ their boundaries in $X, X^{*}, X^{* *}$, respectively. Denote by $\sigma\left(X, X^{*}\right), \sigma\left(X^{*} ; X\right)$ the weak and weak ${ }^{*}$ topologies on $X, X^{*}$, respectively and by $\tau: X \rightarrow X^{* *}$ a canonical mapping of $X$ into $X^{* *}$. We use the notion of rotundity (or strict convexity) of spaces in usual sense. A normed linear space $X$ is said to be:

(i) smooth at $u \in S_{1}(0)$ if its norm $\|\cdot\|$ is Gâteaux differentiable at $u$;

(ii) smooth, if the norm of $X$ is Gâteaux differentiable on $S_{1}(0)$;

(iii) an (F)-space, if its norm is Fréchet differentiable on $S_{1}(0)$;

(iv) weakly locally uniformly rotund (WLUR) at $u \in S_{1}(0)$ if for every sequence $\left(u_{n}\right)$ $\subset S_{1}(0)$ with $\left\|u_{n}+u\right\| \rightarrow 2$ there is $u_{n} \rightarrow u$ in the $\sigma\left(X, X^{*}\right)$-topology;

(v) WLUR at the points of some subset $Q$ of $S_{1}(0)$, if $X$ is WLUR at each point $u$ of $Q$;

(vi) an (h)-space if for each sequence $\left(u_{n}\right)$ in $X$ converging in the $\sigma\left(X, X^{*}\right)$ topology of $X$. to $u_{0}$ and $\left\|u_{n}\right\| \rightarrow\left\|u_{0}\right\|$ we have that $u_{n} \rightarrow u_{0}$ in the norm topology of $X$.

By the gauge function $\mu: \mathbf{R}^{+} \rightarrow \mathbf{R}^{+}$we meān a real valued strictly increasing continuous function such that $\mu(0)=0$ and $\lim _{t \rightarrow \infty} \mu(t)=+\infty$. A set-valued mapping $J: X \rightarrow 2^{X^{\bullet}}$ is said to be a duality mapping of $X$ into $X^{*}$ with the gauge function $\mu$ if $J(0)=\{0\}$ and for each $u \in X, u \neq 0$,

$$
J(u)=\left\{u^{*} \in X^{*}:\left\langle u^{*}, u\right\rangle=\left\|u^{*}\right\| \cdot\|\dot{u}\|,\left\|u^{*}\right\|=\mu(\|u\|)\right\}
$$

For $u \in X, J(u)$ is non-empty convex and $\sigma\left(X^{*}, X\right)$-compact subset of $X^{*}$. $X$ is smooth at $u \in S_{1}(0)$ if and only if $J$ is single-valued at $u$ (see [4]). The duality mapping of $X^{*}$ into $X^{* *}$ is denoted by $J^{*}$ and the duality map $J^{*}: X \rightarrow 2^{X^{* *}}$ with the gauge function $\mu^{*}=\mu^{-1}$ is called the associated duality map with $J$ (see [23]) and we denote it by $J_{\mathrm{a}}^{*}$. By normalized duality mapping we mean the duality mapping with gauge function $\mu(s)=s$. Let $t$ denote the $\sigma\left(X^{*}, X\right), \sigma\left(X, X^{*}\right)$, or norn topology on $X^{*}$. The duality mapping $J: X \rightarrow 2^{X^{*}}$ is said to be upper-semicontinuous at $u_{0} \in X$ from the norm topology of $X$ to the $t$-topology of $X^{*}$ if for any $t$-open set $V$ in $X^{*}$ with $J\left(u_{0}\right) \subset V$ there exists an open neighborhood $U$ of $u_{0}$ such that $J(U) \subset V$. We shall use the following result of SMULian [26]. Let $X$ be a Banach space, then the norm of $X$ is Gâteaux (Fréchet) differentiable at $u_{0} \in S_{1}(0)$ if and only of the following implication holds: when $\left(u_{n}{ }^{*}\right) \subset S_{1}{ }^{*}(0),\left\langle u_{n}{ }^{*}, u_{0}\right\rangle \rightarrow\left\|u_{0}\right\|=1$, then $\left(u_{n}{ }^{*}\right)$ is a weak ${ }^{*}$ (strong) Cauchy sequence. Similarly, necessary and sufficient condition for the fact that the norm of $X^{*}$ is Gâteaux (Fréchet) differentiable at $u_{0}^{*} \in S_{1}^{*}(0)$ is that the following implication is valid: if $\left(u_{n}\right) \subset S_{1}(0),\left\langle u_{0}^{*}, u_{n}\right\rangle \rightarrow 1$, then $\left(u_{n}\right)$ is a weak (strong) Cauchy sequence in $X$.

\section{Some properties of duality mapping}

We shall use the following result, which is a special case of the more general statement proved by Gues [9] (compare also [4, 11]) on the base of the BISHOP-Phelps [3] theorem.

Lem m a 1 : If $X$ is a Banach space and $X^{*}$ is an (F)-space, then $X$ is reflexive.

Proof: Let $u_{0}^{*} \in S_{1}^{*}(0)$ be arbitrary. We show that there exists $u_{0} \in S_{1}(0)$ such that $\left\langle u^{*}, u_{0}\right\rangle=1$. Choose a sequence $\left(u_{n}\right) \subset S_{1}(0)$ such that $\left\langle u_{0}^{*}, u_{n}\right\rangle \rightarrow 1$. By the 
Šmuljan theorem $\left(u_{n}\right)$ is a Cauchy sequence in $X$. Hence $u_{n} \rightarrow u_{0}$ and $u_{0} \in S_{1}(0)$, while $\left\langle u_{0}^{*}, u_{0}\right\rangle=1$. By the JaMEs [17] characterization of reflexivity $X$ is reflexive

- L'em ma 2: Let $X$ be a Banach spàce. Then the jollowing statements are valid:

(i) 'Gnes [10]). A duality mapping $J$ is an homeomorphism of $X$ onto $X^{*}$ if and only if $X$ and $X^{*}$ are both (F)-space, then $X$ is an.(F)-spaces.

(ii) If $X$ is smooth reflexive and $X^{*}$ is an (h)-space, then $X^{\prime}$-is an (F)-space.

Proof: We give it for the sake of completeness.

(i) In comparison with [10] we use here a slight different argument. Assume that $X, X^{*}$ are both $(\mathrm{F})$-spaces. By Lemma $1 X$ is reflexive. Moreover, $J$ is one-to-one, onto and $J, J^{-1}$ are continuous. Conversely, assume that $J$ is a homeomorphism of $X$ onto $X^{*}$. Then $X$ is reflexive and $X$ is an (F)-space. Since $J_{\mathrm{a}}{ }^{*}=\tau J^{-1}$ (see [23]) and $J_{\mathrm{a}}^{*}$ is continuous, $J^{*}$ is also continuous from $X^{*}$ into $X^{* *}$. Hence $X^{*}$ is an $(\mathrm{F})$ space.

(ii) It is sufficient to show that $J$ is continuous on $S_{1}(0)$. Let $u_{0} \in S_{1}(0),\left(u_{n}\right) \subset X$, $u_{n} \rightarrow u_{0}$. Then $\mu\left(\left\|u_{n}\right\|\right) \rightarrow \mu\left(\left\|u_{0}\right\|\right)$ and hence $\left\|J\left(u_{n}\right)\right\| \rightarrow\left\|J\left(u_{0}\right)\right\|$ as $n \rightarrow \infty$. Since $X$ is smooth and reflexive, $J$ is single-valued and continuous from the norm topology. of $X$ to the $\sigma\left(X, X^{*}\right)$-topology of $X^{*}$. As: $X^{*}$ is an (h)-space we conclude that $J\left(u_{n}\right)$ $\rightarrow J\left(u_{0}\right)$ in the norm topology of $X^{*}$

Theorem 1: Let $X$ be a reflexive smooth (h)-Banach space. Then the following statements are valid:

(i) $J$ is a proper map (i.e. for each compact set $G$ of $X^{*}$ the set $J^{-1}(G)$ is compact in $X)$. In addition, if $X$ is rotund, then $J^{-1}$ is continuous on $X^{*}$.

(ii) If $J$ is a local homeomorphism of $X$ into $X^{*}$, then $J$ is a homeomorphism of. $X$ onto $X^{*}$.

(iii) If $X^{*}$, is smooth and (h)-space, then $J$ is a homeomorphism of $X$ onto $X^{*}$.

Proof: It is sufficient to prove our assertions for normalized duality mapping.

(i) Since $X$ is smooth, $J$ is singlevalued on $X$. Reflexivity of $X$ implies that $J$ is onto. Indeed, each $u^{*} \epsilon X^{*}$ is $\sigma\left(X, X^{*}\right)$-continuous and $B_{1}(0)$ is $\sigma\left(X, X^{*}\right)$-compact. By the Weierstrass Theorem each $u^{*} \in X^{*}$ attains its supremum on $S_{1}(0)$. Hence $J(X) \doteq X^{*}$. Let $G$ be a compact subset of $X^{*}$. 'Take $\left(u_{n}\right) \subset J^{-1}(G)$ and set $u_{n}{ }^{*}$ $=J\left(u_{n}\right)$. Then $\left(u_{n}{ }^{*}\right) \subset G$ and in view of the compactness of $G$ in $X^{*}$ there exist a point $u^{*} \in G$ and a subsequence of $\left(u_{n}^{*}\right)$, say $\left(u_{n}^{*}\right)$, such that $u_{n}^{*} \rightarrow u^{*}$. From $\left\|J\left(u_{n}\right)\right\|$ $=\left\|u_{n}^{*}\right\|$ we conclude that $\left(u_{n}\right)$ is bounded in $X$. Hence there exist $u_{0} \in X$ and a subsequence of $\left(u_{n}\right)$, say $\left(u_{n_{1}}\right)$ such that $u_{n} \rightarrow u_{0}$ in the $\sigma\left(X, X^{*}\right)$-topology of $X$. As $\left\|u_{n}^{*}\right\|$ $\rightarrow\left\|u^{*}\right\|$ we have that $\lim \left\|u_{n_{j}}\right\|=\left\|u^{*}\right\|$. Furthermore,

$$
\begin{aligned}
& \left\|u_{0}\right\| \leqq \lim _{j}\left\|u_{n}\right\|=\lim _{j}\left\|u_{n_{j}}\right\|=\lim _{j}\left\|J\left(u_{n}\right)\right\|=\left\|u^{*}\right\| \\
& \stackrel{\lim }{j}\left\|u_{n_{j}}\right\|^{2}=\lim _{j}\left\langle J\left(u_{n_{j}}\right), u_{n_{j}}\right\rangle=\left\langle u^{*}, u_{0}\right\rangle .
\end{aligned}
$$

Hence $\left\|u^{*}\right\|^{2}=\left\langle u^{*}, u_{0}\right\rangle \leqq\left\|u^{*}\right\| \cdot\left\|u_{0}\right\|$, which implies together with the first inequality that $\left\|u^{*}\right\|=\left\|u_{0}\right\|$ and $\left\langle u^{*}, u_{0}\right\rangle=\left\|u_{0}\right\| \cdot\left\|u^{*}\right\|$. Since $J$ is single-valued, $u^{*}=J\left(u_{0}\right)$ and therefore $u_{0} \in J^{-1}(G)$. Moreover, $u_{n} \rightarrow u_{0}$ in the $\sigma\left(X, X^{*}\right)$-topology of $X$ and $\left\|u_{n},\right\|$ $\rightarrow\left\|u^{*}\right\|=\left\|u_{0}\right\|$. As $X$ is an (h)-space, we get thát $u_{n,} \rightarrow u_{0}$, which proves that $J^{-1}(G)$ is compact in $X$ :

In addition, assume that $X$ is rotund. Then $J$ is onto and one-to-one by reflexivity and rotundity of $X$. Let $\left(u_{n}{ }^{*}\right) \subset X^{*}, u_{0}{ }^{*} \in X^{*}, u_{n}{ }^{*} \rightarrow u_{0}^{*}$ in $X^{*}$. From the previous considerations it follows that each subsequence $\left(u_{n_{1}}\right)$ of $\left(u_{n}\right)$, where $u_{n}=J^{-1}\left(u_{n}^{*}\right)$, contains a subsequence converging to $u_{0}=J^{-1}\left(u_{0}^{*}\right)$ in the $\sigma\left(X, X^{*}\right)$-topology of $X$. 
Hence $u_{n} \rightarrow u_{0}$ in the $\sigma\left(X, X^{*}\right)$-topology of $X$ and $\left\|u_{n}\right\| \rightarrow\left\|u_{0}\right\|$. Therefore $u_{n} \rightarrow u_{0}$; which proves the continuity of $J^{-1}$.

(ii) $J$ is proper by (i). Since $J$ is a local homeomorphism of $X$ into $X^{*}$, by the $\mathrm{Ba}$ nach-Mazur theorem (see [24]) $J$ is a global homeomorphism of $X$ onto $X^{*}$.

(iii) This assertion follows at once from Tuemma 2 I

Note that under stronger assumptions on $X, X^{*}$ it was proved in [6], that $J$ is a proper map.

Among others, Yorke [27] proved the following assertions:

(i) If a Banach space $X$ is WLUR at $x \in S_{1}(0)$, then $X^{*}$ is smooth at the points of $J(x) \subset S_{1}(0)$.

(ii) If $X$ is WLUR at some $x^{*} \in J(x)$, then $X$ is very smooth at $x$ (i.e. every support mapping on $X$ is norm to $\sigma\left(X^{*}, X^{* *}\right)$ continuous at $\left.x\right)$. In fact, these results are given in Theorem 2(i) where a different proof method is used.

Let $X$ be a Banach space, $G$ a subset of $S_{1}(0)$ of all functionals of $S_{1}^{*}(0)$ which attains its norm. on $S_{1}(0)$. By the Bisho'p-Phelps theorem $G$ is norm-dense in $S_{1}{ }^{*}(0)$. For each $u^{*} \in G$ there is some $u \in S_{1}(0)$ such that $\left\langle u^{*}, u\right\rangle=1$. Denote by $Q$ the set of such points $u \in S_{1}(0)$ having the above property, where in $Q$ is included just one point $u$ for each $u^{*} \in G$. In next we can assume without loss of generality that $J, J^{*}$ are normalized duality mappings on $X, X^{*}$, respectively.

Theorem 2: Let $X$ be a Banach space. Then the following conclusions are valid:

(i) If $X$ is WLUR at the points of $Q \subset S_{1}(0)$, then $J^{*}$ is single-valued at the points of dense set $G$ of $S_{1}^{*}(0)$. In addition, if $X^{* *}$ is WLUR at the points of $J^{*}(G) \subset S_{1}^{* *}(0)$, then $J^{*}$ is upper-semicontinuous at the points of G from the norm topology of $X^{*}$ to the $\sigma\left(X^{* *}, X^{* * *}\right)$-lopology of $X^{* *}$.

(ii) If $J^{*}$ is single-valued and upper-semicontinuous at the points of $G$ when $X^{*}, X^{* *}$ have the norm topologies, then the strong and weak convergence of sequences of $S_{1}(0)$ coincide at the points of $S_{1}(\dot{0})$. Conversely, if the last condition is satisfied and $X$ is sequentially $\sigma\left(X, X^{*}\right)$-complete and $J^{*}$ is single-valued at the points of $G$, then. $J^{*}$ is upper-semicontinuous at the points of $G$ when $X^{*}$, and $X^{* *}$ have the norm topologies.

Proof: (i) It is sufficient to show that the norm of $X^{*}$ is smooth at the points of dense set $G$ in $S_{1}^{*}(0)$. Tet $u_{0}^{*} \in G$ be arbitrary. Then there exists a point $u_{0} \in Q$ $\subset S_{1}(0)$ such that $\left\langle u_{0}{ }^{*}, u_{0}\right\rangle=\left\|u_{0}^{*}\right\|^{-}=1$. There exists a sequence $\left(u_{n}\right) \subset S_{1}(0)$ such that $\left\langle u_{0}{ }^{*}, u_{n}\right\rangle \rightarrow\left\langle u_{0}^{*}, u_{0}\right\rangle=1$. Then $2 \geqq\left\|u_{n}+u_{0}^{\prime}\right\|=\left\langle u_{0}^{*}, u_{0}+u_{n}\right\rangle$. Since $\left\langle u_{0}^{*}, u_{0}+u_{n}\right\rangle$ $\rightarrow 2$ as $n \rightarrow \infty$, we have that $\left\|u_{0}+u_{n}\right\| \rightarrow 2$. As $X$ is WLUR at the points of $Q$, we conclude that $u_{n}^{\prime} \rightarrow u_{0}$ in the $\sigma\left(X, X^{*}\right)$-topology of $X$. By the Smuljan theorem $X^{*}$ is smooth at $u_{0}^{*}$.

Suppose, in addition, that $X^{* *}$ is WI,UR at the points of the set $J^{*}(G) \subset S_{1}^{* *}(0)$. Let $u_{0}^{*} \in G,\left(u_{n}^{*}\right) \subset X^{*}, u_{n}{ }^{*} \rightarrow u_{0}{ }^{*}$. Without loss of generality one may assume that $u_{n}{ }^{*} \in S_{1}^{*}(0)$. Assume that $v_{n}{ }^{* *} \in J^{*}\left(u_{n}^{*}\right), n=1,2, \ldots$ By (i) $J^{*}$ is single-ralued at $u_{0}^{*}$. Put $v_{0}^{* *}=J^{*}\left(u_{0}^{*}\right)$, it is sufficient to prove that $v_{n}^{* *} \rightarrow v_{0}^{* *}$ in the $\sigma\left(X^{* *}, X^{* * *}\right)$ topology of $X^{* *}$. The properties of $J^{*}$ imply that

Hence

$$
2\left(\left\|u_{n}^{*}\right\|^{2}+\left\|u_{0}^{*}\right\|^{2}\right)-\left\langle v_{n}^{* *}-v_{0}^{* *}, u_{n}^{*}-u_{0}^{*}\right\rangle=\left\langle v_{n}^{* *}+v_{0}^{* *}, u_{0}^{*}+u_{n}^{*}\right\rangle
$$

$$
\begin{aligned}
& 4-\left\|v_{n}^{* *}-v_{0}^{* *}\right\|\left\|u_{n}^{*}-u_{0}^{*}\right\| \leqq 2\left\|v_{n}^{* *}+v_{0}^{* *}\right\| \\
&, \quad . \leqq 2\left(\left\|v_{n}^{* *}\right\|+\left\|v_{0}^{* *}\right\|\right)=4 .
\end{aligned}
$$

Therefore $\left\|v_{n}{ }^{* *}+v_{0}^{* *}\right\| \rightarrow 2$ as $n \rightarrow \infty$. Since $X^{* *}$ is WLUR at the points of $J^{*}(G)$, $v_{n}^{* *} \rightarrow v_{0}^{* *}$ in the $\sigma\left(X^{* *}, X^{* * *}\right)$-topology of $X^{* *}$, which proves that $J^{*}$ is upper- 
semicontinuous at $u_{0}^{*} \in G$ from the norm topology of,$X^{*}$ in the $\sigma\left(X^{* *}, X^{* * *}\right)$-topology of $X^{* *}$.

(ii) If $J^{*}$ is single-valued and upper-semicontinuous at the points of $G \subset S_{1}^{*}(0)$ when $X^{*}, X^{* *}$ have the norm topologies, then the norm of $X^{*}$ is Fréchet-differentiable at the points of $G$ (see [11,13]). Let $u_{0} \in S_{1}(0),\left(u_{u}\right) \subset S_{1}(0), u_{n} \rightarrow u_{0}$ in the $\sigma\left(X, X^{*}\right)$-topology of $X$. Then there exists $u_{0}{ }^{*} \in S_{1}{ }^{*}(0)$ such that $\left\langle u_{0}{ }^{*}, u_{0}\right\rangle=\left\|u_{0}\right\|=1$. Hence $u_{0}^{*} \in G$. Since $\left\langle u_{0}^{*}, u_{n}\right\rangle \rightarrow\left\langle u_{0}^{*}, u_{0}\right\rangle=1$ and the norm of $X^{*}$ is Fréchet-differentiable at the points of $G$, according to the Smuljan theorem $\left(u_{n}\right)$ converges to $u_{0}$ in the norm topology of $X$. Conversely, assume that $X$ is sequentially $\sigma\left(X, X^{*}\right)$ complete and that $J^{*}$ is single-valued at the points of $G$. Then $X^{*}$ is smooth at these points. Let $u_{0}^{*} \in G$ be arbitrary, $\left(u_{n}\right) \subset S_{1}(0)$ be such that $\left\langle u_{0}^{*}, u_{n}\right\rangle \rightarrow 1$. By the Smuljan theorem $\left(u_{n}\right)$ is a weak Cauchy sequence in $X$. Hence $u_{n} \rightarrow u_{0}$ in the $\sigma\left(X, X^{*}\right)$ topology of $X$ for some $u_{0} \in X$. Clearly, $u_{0} \in S_{1}(0)$. According to our hypothesis $u_{n} \rightarrow u_{0}$ in the norm of $X$. Again, in view of the Smuljan theorem, the norm of $X^{*}$ is Fréchet-differentiable at $u_{0}^{*}$. Hence $J$ is upper-semicontinuous at $u_{0}^{*}$ when $X^{*}$ and $X^{* *}$, have the norm topologies (see, $\left.[11-13]\right)$

We shall use the following

Lemma.3 [23]: Let $X$ be a real normed linear space, $J: X \rightarrow 2^{X^{*}}, J^{*}: X^{*} \rightarrow 2^{X^{* \bullet}}$ normalized duality mappings. Then an element $u^{*} \in X^{*}$ lies in $J(u)$ for some $u \in X$ if and only if $\tau(u) \in J^{*}\left(u^{*}\right)$.

Proposition $1:$ Let $X$ be a non-reflexive normed linear space such that $X^{*}$ is smooth, $J: X \rightarrow 2^{X^{*}}, J^{*}: X^{*} \rightarrow X^{* *}$ duality mappings. If $R(J)=X^{*}$, then there exists a separable closed linear subspace $W$ of $X^{*}$ such that $J^{*}$ is not onto $W^{*}$.

Proof: It depends on the arguments of [23] and [5] and it is given here for the sake of completeness. First of all, we show that if $X^{*}$ is smooth and $R(J)=X^{*}$, then $R\left(J^{*}\right)=\tau(X)$. Clearly, $\tau(X) \subset R\left(J^{*}\right)$. Indeed, if $u_{0}^{* *} \in \tau(X)$, then $u_{0}^{* *}$ is $\sigma\left(X^{*}, X\right)-$ continuous on $X^{*}$. Since $B_{1}^{*}(0)$ is $\sigma\left(X^{*}, X\right)$-compact, by the Weierstrass theorem $u_{0}^{* *}$ attains its supremum on $S_{1}^{*}(0)$. Therefore $u_{0}^{* *} \in R\left(J^{*}\right)$. We assert that $R\left(J^{*}\right)$ $\subset \tau(X)$. Assume that $u_{0}^{* *} \in R\left(J^{*}\right), u_{0}^{* *} \neq 0$. Then there is $u_{0}^{*} \in X^{*}$ such that $u_{0}^{* *}$ $=J^{*}\left(u_{0}^{*}\right)$. As $R(J)=X^{*}$ there exists $u_{0}^{*} \in X^{*}$ such that $u_{0}^{*} \in J\left(u_{0}\right)$. By Lemma 3 $\tau\left(u_{0}\right)=J^{*}\left(u_{0}^{*}\right)$. Since $X^{*}$ is smooth we get that $\tau\left(u_{0}\right)=u_{0}^{* *}$ and hence $\tau(X)$ $\supset R\left(J^{*}\right)$. According to our hypothesis $X$ is not reflexive. The ball $B_{1}^{*}(0)$ of $X^{*}$ is $\sigma\left(X^{*}, X\right)$ compact but it is not $\sigma\left(X^{*}, X^{* *}\right)$-countably compact. Indeed,' if $B_{1}^{*}(0)$ would be $\sigma\left(X^{*}, X^{* *}\right)$-countably compact, then $B_{1}^{*}(0)$ would be also $\sigma\left(X^{*}, X^{* *}\right)$-compaict by the Eberlein-Smuljan theorem, which is impossible, because the $\sigma\left(X^{*}, X^{* *}\right)$ - and the $\sigma\left(X^{*}, X\right)$-topologies agree on $X^{*}$ if and only if $X$ is reflexive. As $B_{1}^{*}(0)$ is not $\sigma\left(X^{*}, X^{* *}\right)$-countably compact there is a sequence $\left(u_{n}{ }^{*}\right) \subset B_{1}{ }^{*}(0)$, having no $\sigma\left(X^{*}\right.$, $\left.X^{* *}\right)$-convergent subnet. Since, $B_{1}^{*}(0)$ is $\sigma\left(X^{*}, X\right)$-compact, there is a subnet $\left(u_{a}^{*}\right)$ $\subset\left(u_{n}^{*}\right)$ and a point $u^{*} \in B_{1}^{*}(0)$ such that $u_{\alpha}^{*} \rightarrow u^{*}$ in the $\sigma\left(X^{*}, X\right)$-topology. Put $W=\overline{\text { span }}\left\{\left(u_{n}^{*}\right) \cup\left(u^{*}\right)\right\}$. Then $W$ is closed separable subspace of $X^{*}$. For each fixed $u \in X \tau(u)$ is a $\sigma\left(X^{*}, X\right)$-continuous linear functional on $X^{*}$ and therefore $\langle\tau(u)$, $\left.u_{\alpha}^{*}\right\rangle \rightarrow\left\langle\tau(u), u^{*}\right\rangle$ for each (fixed) $u \in X$. Since $\left(u_{\alpha}^{*}\right)$ is a subnet of $\left(u_{n}^{*}\right)$ and $\left(u_{n}^{*}\right)$ contains no $\sigma\left(X^{*}, X^{* *}\right)$-convergent subnet, we conclude that $u_{\alpha}^{*} \rightarrow u^{*}$ in the $\sigma\left(X^{*}, X^{* *}\right)$-topology of $X^{*}$. Each $z^{* *} \in W^{*}$ is a restriction of some $u^{* *}$ of $X^{* *}$ to $W$ and conversely, each linear continuous functional $z^{* *}$ defined on $W$ can be continuously extended on the whole space $X^{*}$. Hence there is $z^{* *} \in W^{*}$ such that $\left\langle z^{* *}\right.$, $\left.u_{a}^{*}\right\rangle \rightarrow\left\langle z^{* *}, u^{*}\right\rangle$. Thus $z^{* *}$ is not $\sigma\left(X^{*}, X\right)$-continuous, i.e. $z^{* *} \notin \tau(X)=R\left(J^{*}\right)$, which proves the assertion 
Proposition 2: Let $X$ be a normed linear space such that $X^{*}$ is smooth, $M$ a bounded $\sigma\left(X, X^{*}\right)$-closed subset of $X . \dot{I} f R(J)=X^{*}$ and $J^{*}$ is continuous from the $\sigma\left(X^{*}, X\right)$ topology of. $X^{*}$ to the $\sigma\left(X^{* *}, X^{*}\right)$-topology of $X^{* *}$, then $J(M)$ is $\sigma\left(X^{*}, X\right)$-compact.

Proof: Since $M$ is bounded we have that $\sup \{\|J(u)\|: u \in M\}<+\infty$. Hence

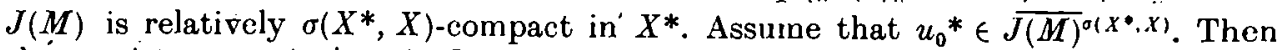
there exists a net $\left(u_{\alpha}{ }^{*}\right)_{\alpha \in I}$ in $J(M)$ such that $u_{\alpha}{ }^{*} \rightarrow u_{0}{ }^{*}$ in the $\sigma\left(X^{*}, X\right)$-topology of $X^{*}$. Hence there are $u_{a} \in M$ such that $u_{a}^{*} \in J\left(u_{a}\right)$ for each $\alpha \in I$. By our hypotheses $J$ is single-valued and $R\left(J^{*}\right)=\tau(X)$ (see the first part of the proof of Proposition 2). In virtue of Lemma $3 \tau\left(u_{\alpha}\right)=J^{*}\left(u_{s}^{*}\right)$ for each $\alpha \in I$ and $J^{*}\left(u_{\alpha}^{*}\right) \rightarrow J^{*}\left(u_{0}^{*}\right)$ in the $\sigma\left(X^{* *}, X^{*}\right)$-topology of $X^{* *}$. Setting $u_{0}^{* *}=J^{*}\left(u_{0}^{*}\right)$ we have that $u_{0}^{* *} \epsilon^{*} \tau(X)$ and hence $u_{0}^{* *}$ is $\sigma\left(X^{*}, X\right)$-continuous on $X^{*}$. There exists a point $u_{0} \in X$ such that $u_{0}^{* *}=\tau\left(u_{0}\right)$. Now we have that $\tau\left(u_{\alpha}\right) \rightarrow \tau\left(u_{0}\right)$ in the $\sigma\left(X^{* *}, X^{*}\right)$-topology of $X^{* *}$. Since $\tau$ is a linear homeomorphism from the space $\left(X, \sigma\left(X, X^{*}\right)\right)$ into the space $\left(X^{* *}, \sigma\left(X^{* *}, X^{*}\right)\right)$, we conclude that $u_{s} \rightarrow u_{0}$ in the $\sigma\left(X, X^{*}\right)$-topology of $X$. Since $M$ is $\sigma\left(X, X^{*}\right)$-closed, $u_{0} \in M$. Because $\tau\left(u_{0}\right)=J^{*}\left(u_{0}^{*}\right)$, by Lemma 3 we get that $u_{0}^{*} \in J\left(u_{0}\right) \subset J(M)$, whịch concludes the proof

\section{The Lax-Milgram property of bilinear forms}

In [20-22] we established some characterizations of reflexivity of Banach spaces by means of the duality mapping. Now we derive once more characterization of reflexivity of Banach spaces based on the so-called Lax-Milgram property of bilinear forms. This result is inspired by $[15,25]$.

Definition 1: Let $X, Y$ be normed linear spaces. We shall say that $X$ has the Lax-Milgram property (LMP) with respect to $Y$ if there exists a bilinear form $Q \times Y \rightarrow \mathbf{C}$ with the following property: For a given linear closed separable subspace $F$ of $X$ there exists a separable subspace $P$ of $Y$ such that $Q$ is bounded on $F \times P$ and for each $u^{*} \in F^{*}$. there exists a unique point $v_{P}$ in $P$ such that $\left\langle u^{*}, u\right\rangle=Q\left(u, v_{P}\right)$ for each $u \in F$.

Sinilarly. we shall say that $Y$ has the LMP with respect to $X$ if there is a bilinear form $Q: X \times Y-\mathrm{C}$ with the following property: For a given linear closed separable subspace $V$ of $Y$ there exists a separable linear subspace $E$ of $X$ such that $Q$ is bounded on $E^{*} \times V$ and for each $v^{*} \in V^{*}$ there exists a unique element $u_{E}$ in $E$ such that $\left\langle v^{*}, v\right\rangle=Q\left(u_{E}, v\right)$ for each $v \in V$. .

Proposition 3: Let $X, Y$ be normed linear spaces. If $X$ is sequentially weakly complete and has the LMP with respect to $Y$, then $X$ is reflexive.

Proof: It relies on the argument of [25]. Let $\left(u_{n}\right)$ be a bounded sequence in $X$. Put $F^{\prime}=\overline{\operatorname{span}}\left\{\left(u_{n}\right)\right\}$. Then $F$ is a closed separable subspace of $X$., By our hypothesis there exist a separable linear subspace $P$ of $Y$ and a bilinear form $Q: X \times Y \rightarrow \mathbf{C}$ such that $Q$ is bounded on $F \times P$, i.e. $|Q(u, v)| \leqq M_{F, P}\|u\| \cdot\|v\|$ for each $u \in F, v \in P^{\text {P }}$. and some constant $M_{r, P}>0$, and the representation of the elements $u^{*} \in F^{*}$ by means of $Q$ and the unique points $v_{P}$ of $P$ is valid. Iet $\left(v_{n}\right)$ be a dense sequence in $P$. Define the linear continúous functionals $\left(u_{n}{ }^{*}\right)$ by $\left\langle u_{n}{ }^{*}, u\right\rangle=Q\left(u, v_{n}\right)$ for each $u \in F$ and $n \cdot(n=1,2, \ldots)$. Clearly, $u_{n}^{*} \in F^{*}$ for each $n$. We assert that $\left(u_{n}^{*}\right)$ is dense in $F^{*}$. Indeed, if $u^{*} \in F^{*}$ is an arbitrary point, then by our hypothesis, there exists a unique point $v_{P} \in P$ such that $\left\langle u^{*}, u\right\rangle=Q\left(u, v_{P}\right)$ for each $u \in F$. As $\left(v_{n}^{\prime}\right)$ is dense in $P$. 
there exists a subsequence of $\left(v_{n}\right)$, say $\left(v_{n}\right)$, such that $v_{n} \rightarrow v_{p}$ as $\dot{n} \rightarrow \infty$. Then ,

$$
\left|\left\langle u^{*}-u_{n}^{*}, u\right\rangle\right|=\left|Q\left(u, v_{P}-v_{n}\right)\right| \leqq M_{F, P}\|u\| \cdot\left\|v_{P}-v_{n}\right\|
$$

for each $u \in F$ and

$$
\left\|u^{*}-u_{n}^{*}\right\| \leqq \sup _{\|u\|=1}\left|\left\langle u^{*}-u_{n}^{*}, u\right\rangle\right| \leqq M_{F, P}\left\|v_{P}-v_{n}\right\| .
$$

Hence $\left(u_{n}{ }^{*}\right)$ is dense in $F^{*}$. Now $\left(\left\langle u_{i}^{*}, u_{n}\right\rangle\right)_{n-1}^{\infty}$ is a bounded sequence of reals for each fixed $i(i=1,2, \ldots)$. By the diagonal process one can extract a subsequence $\left(u_{k}\right)$ of $\left(u_{n}\right)$ such that $\left(\left\langle u_{i}^{*}, u_{k}\right\rangle\right)_{k=1}^{\infty}$ is convergent for each $i$. In view, of the density of $\left(u_{n}{ }^{*}\right)$ in $F^{*}$ we conclude that $\left(\left\langle u^{*}, u_{k}\right\rangle\right)$ is convergent for each $u^{*} \in F^{*}$. By the HahnBanach theorem for each $u^{*} \in F^{*}$ there exists some $v^{*} \in X^{*}$ such that $v^{*} \mid F=u^{*}$ and $\left\|u^{*}\right\|=\left\|v^{*}\right\|$. On the other hand each $v^{*} \in X^{*}$ restricted to $F$ is an element of $F^{*}$. Hence $\left(u_{k}\right)$ is the weak Cauchy sequence in $X$. Since $X$ is sequentially $\sigma\left(X, X^{*}\right)$ complete, there exists a point $u_{0} \in X$ such that $u_{k} \rightarrow u_{0}$ in the $\sigma\left(X, X^{*}\right)$-topology of $X$. Hence $X$ is reflexive

Corollary 1: Let $X, Y$ be sequentially weakly complete Banach spaces such that $X$ has the LMIP with respect to $Y$ and $Y$ has the LMP with respect to $X$. Then $X, Y$ are both reflexive.

Problems: (i) It would be interesting to describe the properties of the set $Q$ (see Theorem 2) in connection with the geometric structure of the Banach spaces.

(ii) Definition 1 together with Proposition 4 and Theorem 1 [15] imply the following question: Let $X$ be a Banach space and assume that each closed separable subspace $F$ of $X$ is isomorphic (isometric) to its dual $F^{*}$. What is the geometric structure of the space $X$ ?

\section{REFERENCES}

[1] BAiAaCH, S.: Théorie des opérations lineaires. Warszawa 1932.

[2] Beurisag, A., and A. E. Livingston: A theorem on duality mapping in Banach spaces. Ark'. Mat. 4 (1962), 405-411.

[3] Bishop, E., and R. R. Phelps: A proof that every Banach space is subreflexive.- Bull. . Amer. Math. Soc. 67 (1961), 97-98.

[4] CudiA, D. F.: The geometry of Banach spaces; Smoothness. Trans. Amer. Math. Soc. 110 (1964), $284-314$.

[5] Davis, W. J., and W. B. Jonnsox: A renorming, of nonreflexive Banach spaces. Proc. Amer. Math. Soc. 17 (1973), 486-488.

[6] Ekeland, I., and G. Lfinoura: Generic Fréchet-differentiability and perturbed optimi; zation problems in Banach spaces. Trans. Amer. Math. Soc. \$94 (1976), 193-216.

[7] Fitzpatrick, S. P.: Continuity of nonlinear monotone operators. Proc. Amer. Math. Soc. 62 (1977), 111-116.

[8] Fitzpaticick, S. P.: Monotone operators and dentability. Bull. Austral. Math. Soc. 1s (1978), $77-82$.

[9] Giles, J. R.: On a differentiability condition for reflexivity of a Banach space. J. Austral. Nath. Soc. 12 (1971), 393-396.

[10] Giles, J. R.: A Banach space with support homeomorphism is reflexive. Bull. Austral. Math. Soc. 5 (1971), 187-189.

[11] GiLes, J. R.: On a characterization of differentiability of the norm of a normed linear space. J. Austral. Math. Soc. 12 (1971), 106-114.

[12] GtLes, J. R.: Convex analysis with application in the differentiation of convex functions (Research Notes in Math. 58). London: Pitman (Adv. Publishing Program 10) 1982. ' 
[13] Giles, J. R., Gregory, D. A., and B. Sims: Geometrical implications of upper semicontinuity of the duality mapping on a Banach space. Pícific J. Math. 79 (1978), 99-109.

[14] Gregory, D. A.: Upper semi-continuity of subdifferential mappings. Canad. Math. Bull. $23(1980), 11-19$.

[15] HAYDEN, T.. L.: Representation theorems in reflexive Banach spaces. Math. Z. 1U4 (1968), $405-406$.

[16] Hille, E., and R. S. PhI.Lms: Functional analysis and semi-groups (Amer. Math. Soc. Colloq: Publ. 31) Providence R. I.: Amer. Math. Soc. 1957.

[17] JAMES, R. C.: Characterization of reflexivity. Studia Math. 23 (1964), 205-216.

[18] Kenderov, P. S.: The set-valued monotone mappings are almost everywhere singlevalued. Studia Math. 56 (1976), 199-203.

[19] Kenderov, P. S.: Monotone operators in Asplund spaces. Compt. Rendus de l'Académie bulgare des Sciences 30 (1977), 963-964.

[20] KoLomý, J., and H. D. VIET: On duality mappings and metric gradient. Boll. Un. Mat. Ital. (5) 16-A (1979), 92-99.

[21] Kolosí, J.: Duality mappings and characterization of reflexivity of Banach spaces. Boll. Un. Mat. Ital. (6) 1-B (1982), 275-283.

[22] Коцомү், J.: Duality mápping and geonetry of Banach spaces. In: General Topology and its relations to modern analysis and algebra V. Proc. Fifth Prague Topol. Symp. 1981 (ed.: J. Novík). Berlin: Heldermann Verlag 1982, 435-441.

[23] De Prima, C. R., and W. V. Prtryshyn: Remarks on strict monotonicity and surjectivity on a real normed linear spaces. Math. Z. 123 (1971), 49-55.

[24] Plastock, R.: Homeomorphisms between Banach spaces. Trans. Amer. Math. Soc. 200 (1974), $169-183$.

[25] SAUER, N.: Representation theorems in a reflexive B-space. Math. Z. 93 (1966), $202-205$.

[2̇6] Шмульян, В. Л.: О некоторых геометрических своиствах единичной сферы про.странства тина (B). Матем. сб. 6 (48) (1939), 77-94.

[27] Yorke, A. C.: Differentiability and local rotundity. J. Austral. Math. Soc. 28 (1979), $205-213$.

Manuskripteingang: 01.07.1983; in revidierter Fassung: 22.03. 1984

\section{VERFASSER:}

Prof. Dr. Josef Kolom Ý

Matematický Ústav University Karlovy

CSSR - 18600 Praha 8 - Karlin, Sokolovská 83 